DOI: $10.15393 / \mathrm{j} 3$.art.2020.7470

UDC 517.521, 517.98

Gh. Rahimlou, V. Sadri, R. Ahmadi

\title{
WEIGHTED RIESZ BASES IN G-FUSION FRAMES AND THEIR PERTURBATION
}

\begin{abstract}
In this paper, we introduce orthonormal and Riesz bases for g-fusion frames and show that the weights have basic roles. Next, we prove an effective theorem between frames and g-fusion frames by using an operator. Finally, perturbations of g-fusion frames will be presented.
\end{abstract}

Key words: g-fusion frame, Dual g-fusion frame, gf-complete, gf-orthonormal basis, gf-Riesz basis

2010 Mathematical Subject Classification: Primary 42C15; Secondary 46C99, $41 A 58$

1. Introduction and preliminaries. Bases play a prominent role in discrete frames and their studying can extract interesting properties from the frames. One of the most important types of bases are orthonormal bases and also, as their special case, the Riesz basis. The Riesz basis has been defined in [6] by the image of orthonormal bases with a bounded bijective operator, but for fusion and generalized frames, there are different strategies [4], [14]. In this paper, we transfer some common properties of g-frames to g-fusion frames, which have been defined by authors. Afterwards, orthonormal bases have been equalized in a property with complete sequences and inequalities for the synthesis operator. Sun in [15] introduced a Riesz basis for g-frames by using that property and we will continue his method in section 1 for g-fusion frames. In section 2, we present a useful operator for characterization of these frames. Finally, in section 3, a perturbation of these frames is studied.

Throughout this paper, $H$ and $K$ are separable Hilbert spaces and $\mathcal{B}(H, K)$ is the collection of all bounded linear operators of $H$ into $K$. If $K=H$, then $\mathcal{B}(H, H)$ is denoted by $\mathcal{B}(H)$. Also, $\pi_{V}$ is the orthogonal

(C) Petrozavodsk State University, 2020 
projection from $H$ onto a closed subspace $V \subset H$ and $\left\{H_{j}\right\}_{j \in \mathbb{J}}$ is a sequence of Hilbert spaces, where $\mathbb{I}$ is a subset of $\mathbb{Z}$. It is easy to check that if $u \in \mathcal{B}(H)$ and $V \subset H$ is a closed subspace, then [11]

$$
\pi_{V} u^{*} \pi_{\overline{u V}}=\pi_{V} u^{*}
$$

We define the space $\mathscr{H}_{2}:=\left(\sum_{j \in \mathbb{J}} \oplus H_{j}\right)_{\ell_{2}}$ by

$$
\mathscr{H}_{2}=\left\{\left\{f_{j}\right\}_{j \in \mathbb{J}}: f_{j} \in H_{j}, \sum_{j \in \mathbb{J}}\left\|f_{j}\right\|^{2}<\infty\right\},
$$

with the inner product defined by

$$
\left\langle\left\{f_{j}\right\},\left\{g_{j}\right\}\right\rangle=\sum_{j \in \mathbb{J}}\left\langle f_{j}, g_{j}\right\rangle .
$$

It is clear that $\mathscr{H}_{2}$ is a Hilbert space with pointwise operations.

Definition 1. Let $W=\left\{W_{j}\right\}_{j \in \mathbb{J}}$ be a collection of closed subspaces of $H,\left\{v_{j}\right\}_{j \in \mathbb{J}}$ be a family of weights, i.e., $v_{j}>0$ and $\Lambda_{j} \in \mathcal{B}\left(H, H_{j}\right)$ for each $j \in \mathbb{J}$. We say $\Lambda:=\left(W_{j}, \Lambda_{j}, v_{j}\right)_{j \in \mathbb{J}}$ is a generalized fusion frame (or g-fusion frame) for $H$ if there exists $0<A \leqslant B<\infty$ such that for each $f \in H$

$$
A\|f\|^{2} \leqslant \sum_{j \in \mathbb{J}} v_{j}^{2}\left\|\Lambda_{j} \pi_{W_{j}} f\right\|^{2} \leqslant B\|f\|^{2} .
$$

We call $\Lambda$ a Parseval g-fusion frame if $A=B=1$. When the right-hand side of (2) holds, $\Lambda$ is called a g-fusion Bessel sequence for $H$ with bound $B$. Throughout this paper, $\Lambda$ is a triple $\left(W_{j}, \Lambda_{j}, v_{j}\right)_{j \in \mathbb{J}}$ unless otherwise noted.

When $\Lambda$ is a g-fusion Bessel sequence, then the synthesis and analysis operators in the g-fusion frames are defined by

$$
\begin{array}{rlrl}
T_{\Lambda}: \mathscr{H}_{2} \longrightarrow H, & T_{\Lambda}^{*}: H \longrightarrow \mathscr{H}_{2}, \\
T_{\Lambda}\left(\left\{f_{j}\right\}_{j \in \mathbb{J}}\right) & =\sum_{j \in \mathbb{J}} v_{j} \pi_{W_{j}} \Lambda_{j}^{*} f_{j}, & T_{\Lambda}^{*}(f)=\left\{v_{j} \Lambda_{j} \pi_{W_{j}} f\right\}_{j \in J} .
\end{array}
$$

Thus, the g-fusion frame operator is given by

$$
S_{\Lambda} f=T_{\Lambda} T_{\Lambda}^{*} f=\sum_{j \in \mathbb{J}} v_{j}^{2} \pi_{W_{j}} \Lambda_{j}^{*} \Lambda_{j} \pi_{W_{j}} f
$$


and

$$
\left\langle S_{\Lambda} f, f\right\rangle=\sum_{j \in \mathbb{J}} v_{j}^{2}\left\|\Lambda_{j} \pi_{W_{j}} f\right\|^{2}
$$

Therefore,

$$
A I d_{H} \leqslant S_{\Lambda} \leqslant B I d_{H}
$$

This means that $S_{\Lambda}$ is a bounded, positive, and invertible operator. So, we have the reconstruction formula for any $f \in H$ :

$$
f=\sum_{j \in \mathbb{J}} v_{j}^{2} \pi_{W_{j}} \Lambda_{j}^{*} \Lambda_{j} \pi_{W_{j}} S_{\Lambda}^{-1} f=\sum_{j \in \mathbb{J}} v_{j}^{2} S_{\Lambda}^{-1} \pi_{W_{j}} \Lambda_{j}^{*} \Lambda_{j} \pi_{W_{j}} f
$$

With the same method as in Theorem 3.1.3 and 5.4.1 in [6], we can prove the following results.

Theorem 1. $\Lambda$ is a $g$-fusion Bessel sequence for $H$ with bound $B$ if and only if the operator $T_{\Lambda}$ is a well-defined and bounded operator with $\left\|T_{\lambda}\right\| \leqslant \sqrt{B}$

Theorem 2. $\Lambda$ is a $g$-fusion frame for $H$ if and only if

$$
\begin{gathered}
T_{\Lambda}: \mathscr{H}_{2} \longrightarrow H \\
T_{\Lambda}\left(\left\{f_{j}\right\}_{j \in \mathbb{J}}\right)=\sum_{j \in \mathbb{J}} v_{j} \pi_{W_{j}} \Lambda_{j}^{*} f_{j}
\end{gathered}
$$

is well-defined, bounded, and surjective.

Definition 2. A g-fusion frame $\widetilde{\Lambda}:=\left(S_{\Lambda}^{-1} W_{j}, \Lambda_{j} \pi_{W_{j}} S_{\Lambda}^{-1}, v_{j}\right)_{j \in \mathbb{J}}$ with g-fusion frame operator $S_{\widetilde{\Lambda}}=T_{\widetilde{\Lambda}} T_{\widetilde{\Lambda}}^{*}$ is called the (canonical) dual g-fusion frame of $\Lambda$.

It is easy to check that for each $f \in H$,

$$
T_{\widetilde{\Lambda}}^{*} f=T_{\Lambda}^{*}\left(S_{\Lambda}^{-1} f\right)
$$

Hence, $T_{\Lambda} T_{\widetilde{\Lambda}}^{*}=I d_{H}$ and also $S_{\widetilde{\Lambda}}=S_{\Lambda}^{-1}$. Now, we can obtain, by (??),

$$
f=\sum_{j \in \mathbb{J}} v_{j}^{2} \pi_{W_{j}} \Lambda_{j}^{*} \widetilde{\Lambda_{j}} \pi_{\widetilde{W}_{j}} f=\sum_{j \in \mathbb{J}} v_{j}^{2} \pi_{\widetilde{W}_{j}} \widetilde{\Lambda}_{j}^{*} \Lambda_{j} \pi_{W_{j}} f,
$$

where $\widetilde{W_{j}}:=S_{\Lambda}^{-1} W_{j}$ and $\tilde{\Lambda_{j}}:=\Lambda_{j} \pi_{W_{j}} S_{\Lambda}^{-1}$. 
Definition 3. Let $\Lambda_{j} \in \mathcal{B}\left(H, H_{j}\right)$ for each $j \in \mathbb{J}$. $\Lambda=\left(W_{j}, \Lambda_{j}, v_{j}\right)_{j \in \mathbb{J}}$ is called a $g$-f-complete, if

$$
\overline{\operatorname{span}}\left\{\pi_{W_{j}} \Lambda_{j}^{*} H_{j}\right\}=H .
$$

It is easy to check that $\Lambda$ is g-f-complete if and only if

$$
\left\{f: \Lambda_{j} \pi_{W_{j}} f=0, j \in \mathbb{J}\right\}=\{0\} .
$$

Theorem 3. If $\Lambda=\left(W_{j}, \Lambda_{j}, v_{j}\right)_{j \in \mathbb{J}}$ is a g-fusion frame for $H$, then $\Lambda$ is a g-f-complete.

Proof. Let $f \in\left(\operatorname{span}\left\{\pi_{W_{j}} \Lambda_{j}^{*} H_{j}\right\}\right)^{\perp} \subseteq H$. For each $j \in \mathbb{J}$ and $g_{j} \in H_{j}$, we have

$$
\left\langle\Lambda_{j} \pi_{W_{j}} f, g_{j}\right\rangle=\left\langle f, \pi_{W_{j}} \Lambda_{j}^{*} g_{j}\right\rangle=0,
$$

so, $\Lambda_{j} \pi_{W_{j}} f=0$ for all $j \in \mathbb{J}$. Since $\Lambda$ is a g-fusion frame for $H$, then $\|f\|=0$. Thus, $f=0$, and we get $\left(\operatorname{span}\left\{\pi_{W_{j}} \Lambda_{j}^{*} H_{j}\right\}\right)^{\perp}=\{0\}$.

2. G-f-Riesz and orthonormal bases. In this section, we aim to introduce Riesz and orthonormal bases for g-fusion frames, which are extension cases of g-frames, and present some results about them.

Definition 4. Let $W=\left\{W_{j}\right\}_{j \in \mathbb{J}}$ be a collection of closed subspaces of $H$ and $j \in \mathbb{J}$. We say that $\left(W_{j}, \Lambda_{j}\right)_{j \in \mathbb{J}}$ is a $g$-f-orthonormal bases for $H$ with respect to $\left\{v_{j}\right\}_{j \in \mathbb{J}}$, if

$$
\begin{gathered}
\left\langle v_{i} \pi_{W_{i}} \Lambda_{i}^{*} g_{i}, v_{j} \pi_{W_{j}} \Lambda_{j}^{*} g_{j}\right\rangle=\delta_{i, j}\left\langle g_{i}, g_{j}\right\rangle, \quad i, j \in \mathbb{J}, \quad g_{i} \in H_{i}, \quad g_{j} \in H_{j} \\
\sum_{j \in \mathbb{J}} v_{j}^{2}\left\|\Lambda_{j} \pi_{W_{j}} f\right\|^{2}=\|f\|^{2}, \quad f \in H .
\end{gathered}
$$

Definition 5. $\Lambda=\left(W_{j}, \Lambda_{j}, v_{j}\right)_{j \in \mathbb{J}}$ is called a $g$-f-Riesz basis for $H$ if

1) $\Lambda$ is g-f-complete,

2) There exist $0<A \leqslant B<\infty$, such that for each finite subset $\mathbb{I} \subseteq \mathbb{J}$ and $g_{j} \in H_{j}, j \in \mathbb{I}$,

$$
A \sum_{j \in \mathbb{I}}\left\|g_{j}\right\|^{2} \leqslant\left\|\sum_{j \in \mathbb{I}} v_{j} \pi_{W_{j}} \Lambda_{j}^{*} g_{j}\right\|^{2} \leqslant B \sum_{j \in \mathbb{I}}\left\|g_{j}\right\|^{2} .
$$


It is easy to check that if $\Lambda$ is a g-f-Riesz basis for $H$, then the operator $T_{\Lambda}$ defined by

$$
\begin{aligned}
T_{\Lambda} & : \mathscr{H}_{2} \longrightarrow H, \\
T_{\Lambda}\left(\left\{g_{j}\right\}_{j \in \mathbb{J}}\right) & =\sum_{j \in \mathbb{J}} v_{j} \pi_{W_{j}} \Lambda_{j}^{*} g_{j},
\end{aligned}
$$

is injective.

Theorem 4. Let $\Lambda=\left(W_{j}, \Lambda_{j}, v_{j}\right)_{j \in \mathbb{J}}$ be a g-fusion frame for $H$ and suppose that (6) holds. Then $\Lambda$ is a $g$-f-orthonormal basis for $H$.

Proof. Assume that $S_{\Lambda}$ is the g-fusion frame operator of $\Lambda$ and

$$
M:=\left\{f \in H: S_{\Lambda} f=f\right\} .
$$

It is clear that $M$ is a non-empty closed subspace of $H$. Let $f \in H$ and $k \in \mathbb{J}$. Since

$$
v_{j} v_{k} \pi_{W_{j}} \Lambda_{j}^{*} \Lambda_{j} \pi_{W_{j}} \pi_{W_{k}} \Lambda_{k}^{*} \Lambda_{k} \pi_{W_{k}} f=\delta_{j, k} \pi_{W_{k}} \Lambda_{k}^{*} \Lambda_{k} \pi_{W_{k}} f,
$$

then $\pi_{W_{k}} \Lambda_{k}^{*} \Lambda_{k} \pi_{W_{k}} f \in M$. So, for any $h \in M^{\perp}$ and $g \in M$ we have, for all $j \in \mathbb{J}$

$$
\left\langle\pi_{W_{j}} \Lambda_{j}^{*} \Lambda_{j} \pi_{W_{j}} h, g\right\rangle=\left\langle h, \pi_{W_{j}} \Lambda_{j}^{*} \Lambda_{j} \pi_{W_{j}} g\right\rangle=0 .
$$

Thus, $\pi_{W_{j}} \Lambda_{j}^{*} \Lambda_{j} \pi_{W_{j}} h=0$ for each $j \in \mathbb{J}$, and so $\left\|\Lambda_{j} \pi_{W_{j}} h\right\|=0$. By definition of g-fusion frame, we obtain $h=0$. Therefore, $M^{\perp}=\{0\}$, and we conclude $H=M$. So, $S_{\Lambda}=I d_{H}$, and the proof is completed.

Theorem 5. $\Lambda$ is a $g$-f-orthonormal basis for $H$ if and only if

(I) $v_{j} \pi_{W_{j}} \Lambda_{j}^{*}$ is isometric for any $j \in \mathbb{J}$;

(II) $\bigoplus_{j \in J} v_{j} \pi_{W_{j}} \Lambda_{j}^{*}\left(H_{j}\right)=H$.

Proof. Suppose that $\Lambda$ is a g-f-orthonormal basis for $H$. If $j \in \mathbb{J}$ and $g \in H_{j}$, we have

$$
\left\langle v_{j} \pi_{W_{j}} \Lambda_{j}^{*} g, v_{j} \pi_{W_{j}} \Lambda_{j}^{*} g\right\rangle=\delta_{j, j}\langle g, g\rangle=\langle g, g\rangle .
$$

Then, $v_{j} \pi_{W_{j}} \Lambda_{j}^{*}$ is isometric for any $j \in \mathbb{J}$. So, $v_{j} \pi_{W_{j}} \Lambda_{j}^{*}\left(H_{j}\right)$ is a closed subspace of $H$. Therefore, for each $j \in \mathbb{J}$ and $f \in H$, we have from (7):

$$
\langle f, f\rangle=\sum_{j \in \mathbb{I}} v_{j}^{2}\left\langle\Lambda_{j} \pi_{W_{j}} f, \Lambda_{j} \pi_{W_{j}} f\right\rangle=
$$




$$
=\left\langle\sum_{j \in \mathbb{J}} v_{j}^{2} \pi_{W_{j}} \Lambda_{j}^{*} \Lambda_{j} \pi_{W_{j}} f, f\right\rangle .
$$

Thus, for each $f \in H$,

$$
f=\sum_{j \in \mathbb{I}} v_{j}^{2} \pi_{W_{j}} \Lambda_{j}^{*} \Lambda_{j} \pi_{W_{j}} f
$$

By letting $g_{j}:=v_{j} \Lambda_{j} \pi_{W_{j}} f$, we obtain $f=\sum_{j \in \mathbb{J}} v_{j} \pi_{W_{j}} \Lambda_{j}^{*} g_{j}$ and

$$
\sum_{j \in \mathbb{J}} v_{j}^{2}\left\|\pi_{W_{j}} \Lambda_{j}^{*} g_{j}\right\|^{2}=\sum_{j \in \mathbb{J}}\left\|g_{j}\right\|^{2}=\sum_{j \in \mathbb{J}} v_{j}^{2}\left\|\Lambda_{j} \pi_{W_{j}} f\right\|^{2}=\|f\|^{2} .
$$

So, $\bigoplus_{j \in \mathbb{J}} v_{j} \pi_{W_{j}} \Lambda_{j}^{*}\left(H_{j}\right)=H$. Conversely, if (I), (II) are satisfied, then (6) is clear. Indeed, for each $i \neq j, v_{i} \pi_{W_{i}} \Lambda_{i}^{*}\left(H_{i}\right) \perp v_{j} \pi_{W_{j}} \Lambda_{j}^{*}\left(H_{j}\right)$ and $v_{j} \pi_{W_{j}} \Lambda_{j}^{*}$ are isometric. Let $f \in H$; we get from (II), for any $j \in \mathbb{J}$ and some $g_{j} \in H_{j}$,

$$
f=\sum_{j \in \mathbb{I}} v_{j} \pi_{W_{j}} \Lambda_{j}^{*} g_{j}
$$

and

$$
\|f\|^{2}=\sum_{j \in \mathbb{J}} v_{j}^{2}\left\|\pi_{W_{j}} \Lambda_{j}^{*} g_{j}\right\|^{2}=\sum_{j \in \mathbb{J}}\left\|g_{j}\right\|^{2} .
$$

Now, let $i \in \mathbb{J}$; then, for each $f, h \in H_{j}$,

$$
\begin{aligned}
\left\langle v_{j} \Lambda_{i} \pi_{W_{i}} f, h\right\rangle & =\left\langle\sum_{j \in \mathbb{J}} v_{j}^{2} \Lambda_{i} \pi_{W_{i}} \pi_{W_{j}} \Lambda_{j}^{*} g_{j}, h\right\rangle= \\
& =\sum_{j \in \mathbb{J}} v_{j}^{2}\left\langle\pi_{W_{j}} \Lambda_{j}^{*} g_{j}, \pi_{W_{i}} \Lambda_{i}^{*} h\right\rangle= \\
& =\left\langle v_{i} \pi_{W_{i}} \Lambda_{i}^{*} g_{i}, v_{i} \pi_{W_{i}} \Lambda_{i}^{*} h\right\rangle= \\
& =\left\langle g_{i}, h\right\rangle .
\end{aligned}
$$

Hence, $g_{i}=v_{i} \Lambda_{i} \pi_{W_{i}} f$ for each $i \in \mathbb{J}$. So, $f=\sum_{j \in \mathbb{J}} v_{j}^{2} \pi_{W_{j}} \Lambda_{j}^{*} \Lambda_{j} \pi_{W_{j}} f$ for all $f \in H$, and (7) is proved.

Corollary 1. Every g-f-orthonormal basis for $H$ is a $g$-f-Riesz basis for $H$ with the bounds $A=B=1$.

Theorem 6. Let $\Theta=\left(W_{j}, \Theta_{j}\right)_{j \in \mathbb{J}}$ be a g-f-orthonormal basis with respect to $\left\{v_{j}\right\}_{j \in \mathbb{J}}$ and $\Lambda=\left(W_{j}, \Lambda_{j}, v_{j}\right)_{j \in \mathbb{J}}$ be a $g$-fusion frame for $H$ with 
the same weights. Then there exists a surjective operator $V \in \mathcal{B}(H)$, such that $\Lambda_{j} \pi_{W_{j}}=\Theta_{j} \pi_{W_{j}} V^{*}$ for all $j \in \mathbb{J}$.

Proof. Let

$$
\begin{gathered}
V: H \longrightarrow H, \\
V f=\sum_{j \in \mathbb{J}} v_{j}^{2} \pi_{W_{j}} \Lambda_{j}^{*} \Theta_{j} \pi_{W_{j}} f .
\end{gathered}
$$

Then $V$ is well-defined and bounded. Indeed, for each finite subset $\mathbb{I} \subseteq \mathbb{J}$ and $f \in H$,

$$
\begin{aligned}
\|V f\| & =\sup _{\|h\|=1}\left|\left\langle\sum_{j \in \mathbb{J}} v_{j}^{2} \pi_{W_{j}} \Lambda_{j}^{*} \Theta_{j} \pi_{W_{j}} f, h\right\rangle\right| \leqslant \\
& \leqslant \sqrt{B}\left(\sum_{j \in \mathbb{J}} v_{j}^{2}\left\|\Theta_{j} \pi_{W_{j}} f\right\|^{2}\right)^{\frac{1}{2}}=\sqrt{B}\|f\|,
\end{aligned}
$$

where $B$ is an upper g-fusion frame bound for $\Lambda$. Therefore, the series is weakly unconditionally Cauchy, and so is unconditionally convergent in $H$ (see [7], page 58), and also $\|V\| \leqslant \sqrt{B}$. Since $\Theta$ is a g-f-orthonormal basis, then

$$
\Theta_{j} \pi_{W_{j}} \pi_{W_{i}} \Theta_{i}^{*} g=\left(v_{i} v_{j}\right)^{-1} \delta_{i, j} g
$$

and

$$
V \pi_{W_{i}} \Theta_{i}^{*} g=\sum_{j \in \mathbb{I}} v_{j}^{2} \pi_{W_{j}} \Lambda_{j}^{*} \Theta_{j} \pi_{W_{j}} \pi_{W_{i}} \Theta_{i}^{*} g=\pi_{W_{i}} \Lambda_{i}^{*} g,
$$

for all $g \in H_{j}$ and $i \in \mathbb{J}$. Thus, $\Lambda_{j} \pi_{W_{j}}=\Theta_{j} \pi_{W_{j}} V^{*}$. Now, we show that $V$ is surjective. Assume that $f \in H$. By Theorem 2, there is $\left\{g_{j}\right\}_{j \in \mathbb{J}} \in \mathscr{H}_{2}$, such that $\sum_{j \in \mathbb{J}} v_{j} \pi_{W_{j}} \Lambda_{j}^{*} g_{j}=f$. Let $g:=T_{\Theta}\left(\left\{g_{j}\right\}_{j \in \mathbb{J}}\right)$, thus

$$
V g=\sum_{j \in \mathbb{I}} V v_{j} \pi_{W_{j}} \Theta_{j}^{*} g_{j}=\sum_{j \in \mathbb{J}} v_{j} \pi_{W_{j}} \Lambda_{j}^{*} g_{j}=f
$$

and $V$ is surjective.

Corollary 1. If $\Lambda$ is a Parseval $g$-fusion frame for $H$, then $V^{*}$ is isometric.

Corollary 2. If $\Lambda$ is a g-f-Riesz basis for $H$, then $V$ is invertible.

Proof. Let $V f=0$ and $f \in H$. Since $T_{\Lambda}$ is injective and

$$
V f=\sum_{j \in \mathbb{J}} v_{j}^{2} \pi_{W_{j}} \Lambda_{j}^{*} \Theta_{j} \pi_{W_{j}} f=T_{\Lambda} T_{\Theta}^{*} f
$$


therefore, $T_{\Theta}^{*} f=0$. So, $\|f\|^{2}=\left\|T_{\Theta} f\right\|^{2}=0$, hence, $f=0$.

Corollary 3. If $\left(W_{j}, \Lambda_{j}\right)_{j \in \mathbb{J}}$ is a $g$-f-orthonormal basis for $H$ with respect to $\left\{v_{j}\right\}_{j \in \mathbb{J}}$, then $V$ is unitary.

Proof. By Corollaries 1 and 2, the operator $V$ is invertible. Let $f \in H$. We obtain

$$
\|f\|^{2}=\sum_{j \in \mathbb{J}} v_{j}^{2}\left\|\Lambda_{j} \pi_{W_{j}} f\right\|^{2}=\sum_{j \in \mathbb{J}}\left\|\Theta_{j} \pi_{W_{j}} V^{*} f\right\|^{2}=\left\|V^{*} f\right\|^{2} .
$$

Thus, $V V^{*}=i d_{H}$; this means that $V$ is unitary.

3. Characterizations of g-fusion frames, g-f-Riesz and g-f-orthonormal bases. Sun in [15] showed that each g-frame for $H$ induces a sequence in $H$, dependent on the g-frame; he also proved a useful theorem about them. In this section, we are going to extend Sun's method for g-fusion frames.

Let $W=\left\{W_{j}\right\}_{j \in \mathbb{J}}$ be a family of closed subspaces of $H,\left\{v_{j}\right\}_{j \in \mathbb{J}}$ be a family of weights, $\Lambda_{j} \in \mathcal{B}\left(H, H_{j}\right)$ for each $j \in \mathbb{J}$ and $\left\{e_{j, k}\right\}_{k \in \mathbb{K}_{j}}$ be an orthonormal basis for $H_{j}$, where $\mathbb{K}_{j} \subseteq \mathbb{Z}$ and $j \in \mathbb{J}$. Suppose that

$$
\begin{gathered}
\varphi: h \longrightarrow \mathbb{C}, \\
\varphi(f)=\left\langle v_{j} \Lambda_{j} \pi_{W_{j}} f, e_{j, k}\right\rangle .
\end{gathered}
$$

We have

$$
\|\varphi f\| \leqslant v_{j}\left\|\Lambda_{j}\right\|\|f\|
$$

therefore, $\varphi$ is a bounded linear functional on $H$. Now, we can write

$$
\left\langle v_{j} \Lambda_{j} \pi_{W_{j}} f, e_{j, k}\right\rangle=\left\langle f, v_{j} \pi_{W_{j}} \Lambda_{j}^{*} e_{j, k}\right\rangle .
$$

So, if

$$
u_{j, k}:=v_{j} \pi_{W_{j}} \Lambda_{j}^{*} e_{j, k}, \quad j \in \mathbb{J}, k \in \mathbb{K}_{j}
$$

then $\left\langle f, u_{j, k}\right\rangle=\left\langle v_{j} \Lambda_{j} \pi_{W_{j}} f, e_{j, k}\right\rangle$ for all $f \in H$.

Remark 1. Using (9), we get for each $f \in H$ :

$$
v_{j} \Lambda_{j} \pi_{W_{j}} f=\sum_{k \in \mathbb{K}_{j}}\left\langle f, u_{j, k}\right\rangle e_{j, k} .
$$

But

$$
\sum_{k \in \mathbb{K}_{j}}\left|\left\langle f, u_{j, k}\right\rangle\right|^{2} \leqslant v_{j}^{2}\left\|\Lambda_{j}\right\|^{2}\|f\|^{2} .
$$


Thus, $\left\{u_{j, k}\right\}_{k \in \mathbb{K}_{j}}$ is a Bessel sequence for $H$. It follows that for each $f \in H$ and $g \in H_{j}$ :

$$
\begin{aligned}
\left\langle f, v_{j} \pi_{W_{j}} \Lambda_{j}^{*} g\right\rangle & =\left\langle v_{j} \Lambda_{j} \pi_{W_{j}} f, g\right\rangle=\sum_{k \in \mathbb{K}_{j}}\left\langle v_{j} \pi_{W_{j}} \Lambda_{j} f, e_{j, k}\right\rangle\left\langle e_{j, k}, g\right\rangle= \\
& =\sum_{k \in \mathbb{K}_{j}}\left\langle f, u_{j, k}\right\rangle\left\langle e_{j, k}, g\right\rangle=\left\langle f, \sum_{k \in \mathbb{K}_{j}}\left\langle g, e_{j, k}\right\rangle u_{j, k}\right\rangle .
\end{aligned}
$$

Therefore,

$$
v_{j} \pi_{W_{j}} \Lambda_{j}^{*} g=\sum_{k \in \mathbb{K}_{j}}\left\langle g, e_{j, k}\right\rangle u_{j, k}
$$

for all $g \in H_{j}$.

We call $\left\{u_{j, k}: j \in \mathbb{J}, k \in \mathbb{K}_{j}\right\}$ the sequence induced by $\Lambda$.

Theorem 7. Let $\Lambda=\left(W_{j}, \Lambda_{j}, v_{j}\right)_{j \in \mathbb{J}}$ and $u_{j, k}$ be defined by (9). Then we get the following:

(I) $\Lambda$ is a g-fusion frame (resp. g-fusion Bessel sequence, Parseval gfusion frame, $g$-f-Riesz basis, $g$-f-orthonormal basis) for $H$ if and only if $\left\{u_{j, k}: j \in \mathbb{J}, k \in \mathbb{K}_{j}\right\}$ is a frame (resp. Bessel sequence, Parseval frame, Riesz basis, orthonormal basis) for $H$.

(II) The g-fusion operator for $\Lambda$ coincides with the frame operator for $\left\{u_{j, k}: j \in \mathbb{J}, k \in \mathbb{K}_{j}\right\}$.

Proof. (I). By (10), $\Lambda$ is a g-fusion frame (resp. g-fusion Bessel sequence, Parseval g-fusion frame) for $H$ if and only if $\left\{u_{j, k}: j \in \mathbb{J}, k \in \mathbb{K}_{j}\right\}$ is a frame (resp. Bessel sequence, Parseval frame) for $H$.

Assume that $\Lambda$ is a g-f-Riesz basis for $H$ and $g_{j} \in H_{j}$. Thus,

$$
g_{j}=\sum_{k \in \mathbb{K}_{j}} c_{j, k} e_{j, k}
$$

where $c_{j, k} \in \ell\left(\mathbb{K}_{j}\right)$. Note that, by $(10)$,

$$
\left\{f: \Lambda_{j} \pi_{W_{j}} f=0, \quad j \in \mathbb{J}\right\}=\left\{f:\left\langle f, u_{j, k}\right\rangle=0, \quad j \in \mathbb{J}, k \in \mathbb{K}_{j}\right\}
$$

and

$$
A \sum_{j \in \mathbb{I}}\left\|g_{j}\right\|^{2} \leqslant\left\|\sum_{j \in \mathbb{I}} v_{j} \pi_{W_{j}} \Lambda_{j}^{*} g_{j}\right\|^{2} \leqslant B \sum_{j \in \mathbb{I}}\left\|g_{j}\right\|^{2}
$$


is equivalent to

$$
A \sum_{j \in \mathbb{I}} \sum_{k \in \mathbb{K}_{j}}\left|c_{j, k}\right|^{2} \leqslant\left\|\sum_{j \in \mathbb{I}} \sum_{k \in \mathbb{K}_{j}} c_{j, k} u_{j, k}\right\|^{2} \leqslant B \sum_{j \in \mathbb{I}} \sum_{k \in \mathbb{K}_{j}}\left|c_{j, k}\right|^{2}
$$

for any finite $\mathbb{I} \subseteq \mathbb{J}$. Thus, $\Lambda$ is a g-f-Riesz basis if and only if $\left\{u_{j, k}: j \in J, k \in \mathbb{K}_{j}\right\}$ is a Riesz basis.

Now, let $\left(W_{j}, \Lambda_{j}\right)_{j \in \mathbb{J}}$ be a g-f-orthonormal basis for $H$ with respect to $\left\{v_{j}\right\}_{j \in \mathbb{J}}$. We get, for any $j_{1}, j_{2} \in \mathbb{J}, k_{1} \in \mathbb{K}_{j_{1}}$ and $k_{2} \in \mathbb{K}_{j_{2}}$ :

$$
\left\langle u_{j_{1}, k_{1}}, u_{j_{2}, k_{2}}\right\rangle=\left\langle v_{j_{1}} \pi_{W_{j_{1}}} \Lambda_{j_{1}}^{*} e_{j_{1}, k_{1}}, v_{j_{2}} \pi_{W_{j_{2}}} \Lambda_{j_{2}}^{*} e_{j_{2}, k_{2}}\right\rangle=\delta_{j_{1}, k_{1}} \delta_{j_{2}, k_{2}} .
$$

So, $\left\{u_{j, k}: j \in \mathbb{J}, k \in \mathbb{K}_{j}\right\}$ is an orthonormal sequence. Moreover

$$
\|f\|^{2}=\sum_{j \in \mathbb{J}} v_{j}^{2}\left\|\Lambda_{j} \pi_{W_{j}} f\right\|^{2}=\sum_{j \in \mathbb{J}} \sum_{k \in \mathbb{K}_{j}}\left|\left\langle f, u_{j, k}\right\rangle\right|^{2}
$$

for any $f \in H$. Hence, $\left\{u_{j, k}: j \in \mathbb{J}, k \in \mathbb{K}_{j}\right\}$ is an orthonormal basis. For the opposite implication, we need to prove only that (6) holds. We have, by (11), for each $j_{1} \neq j_{2} \in \mathbb{J}: g_{j_{1}} \in H_{j_{1}}$ and $g_{j_{2}} \in H_{j_{2}}$,

$$
\begin{aligned}
\left\langle v_{j_{1}} \pi_{W_{j_{1}}} \Lambda_{j_{1}}^{*} g_{j_{1}}, v_{j_{2}} \pi_{W_{j_{2}}} \Lambda_{j_{2}}^{*} g_{j_{2}}\right\rangle= & \\
& =\left\langle\sum_{k_{1} \in \mathbb{K}_{j_{1}}}\left\langle g_{j_{1}}, e_{j_{1}, k_{1}}\right\rangle u_{j_{1}, k_{1}}, \sum_{k_{2} \in \mathbb{K}_{j_{2}}}\left\langle g_{j_{2}}, e_{j_{2}, k_{2}}\right\rangle u_{j_{2}, k_{2}}\right\rangle=0
\end{aligned}
$$

and for all $g_{1}, g_{2} \in H_{j}$ :

$$
\begin{aligned}
\left\langle v_{j} \pi_{W_{j}} \Lambda_{j}^{*} g_{1}, v_{j} \pi_{W_{j}} \Lambda_{j}^{*} g_{2}\right\rangle= & \\
& =\left\langle\sum_{k_{1} \in \mathbb{K}_{j}}\left\langle g_{1}, e_{j, k_{1}}\right\rangle u_{j, k_{1}}, \sum_{k_{2} \in \mathbb{K}_{j}}\left\langle g_{2}, e_{j, k_{2}}\right\rangle u_{j, k_{2}}\right\rangle=\left\langle g_{1}, g_{2}\right\rangle .
\end{aligned}
$$

(II). By (10) and (11), we have, for any $f \in H$ :

$$
\begin{aligned}
\sum_{j \in \mathbb{J}} v_{j}^{2} \pi_{W_{j}} \Lambda_{j}^{*} \Lambda_{j} \pi_{W_{j}} f=\sum_{j \in \mathbb{J}} \sum_{k \in \mathbb{K}_{j}}\left\langle v_{j} \Lambda_{j} \pi_{W_{j}} f, e_{j, k}\right\rangle u_{j, k}= \\
=\sum_{j \in \mathbb{J}} \sum_{k \in \mathbb{K}_{j}}\left\langle\sum_{k^{\prime} \in \mathbb{K}_{j}}\left\langle f, u_{j, k^{\prime}} e_{j, k^{\prime}}\right\rangle, e_{j, k}\right\rangle u_{j, k}=\sum_{j \in \mathbb{J}} \sum_{k \in \mathbb{K}_{j}}\left\langle f, u_{j, k}\right\rangle u_{j, k} .
\end{aligned}
$$

Corollary 1. Let $\Lambda=\left(W_{j}, \Lambda_{j}, v_{j}\right)_{j \in \mathbb{J}}$ be a $g$-fusion frame for $H$. Then the following are equivalent: 
(I) $\Lambda$ is a $g$-f-Riesz basis for $H$.

(II) For any finite subset $\mathbb{I} \subset \mathbb{J}$, if $\sum_{j \in \mathbb{I}} v_{j} \pi_{W_{j}} \Lambda_{j}^{*} g_{j}=0$ for some $\left\{g_{j}\right\}_{j \in \mathbb{I}} \in$ $\mathscr{H}_{2}$, then $g_{j}=0$ for all $j \in \mathbb{J}$.

Proof. (I) $\Rightarrow(\mathrm{II})$. Let $\mathbb{I} \subset \mathbb{J}$ be finite. So, by (8), we get $\sum_{j \in \mathbb{I}}\left\|g_{j}\right\|^{2}=0$. Thus, $g_{j}=0$ for all $j \in \mathbb{I}$ and so it is true for all $j \in \mathbb{J}$.

$(\mathrm{II}) \Rightarrow(\mathrm{I})$. Let $j \in \mathbb{J}$ and $\left\{e_{j, k}\right\}_{k \in \mathbb{K}_{j}}$ be an orthonormal basis for $H_{j}$ where $\mathbb{K}_{j} \subset \mathbb{Z}$. Assume that $\left\{g_{j}\right\}_{j \in \mathbb{I}} \in \mathscr{H}_{2}$, such that $\sum_{j \in \mathbb{I}} v_{j} \pi_{W_{j}} \Lambda_{j}^{*} g_{j}=0$. Therefore,

$$
g_{j}=\sum_{k \in \mathbb{K}_{j}}\left\langle g_{j}, e_{j, k}\right\rangle e_{j, k}
$$

Hence,

$$
0=\sum_{j \in \mathbb{I}} v_{j} \pi_{W_{j}} \Lambda_{j}^{*} g_{j}=\sum_{j \in \mathbb{I}} \sum_{k \in \mathbb{K}_{j}}\left\langle g_{j}, e_{j, k}\right\rangle v_{j} \pi_{W_{j}} \Lambda_{j}^{*} e_{j, k} .
$$

Now, if $u_{j, k}:=v_{j} \pi_{W_{j}} \Lambda_{j}^{*} e_{j, k}$, then $\left\langle g_{j}, e_{j, k}\right\rangle=0$ by item (II) for every $j \in \mathbb{J}$ and $k \in \mathbb{K}_{j}$. So, by Theorem 5.2.2 in [6], we state that $\left\{u_{j, k}\right\}_{j \in \mathbb{J}, k \in \mathbb{K}_{j}}$ is a Riesz basis; so, by Theorem $7, \Lambda$ is a g-f-Riesz basis.

4. Perturbation of g-fusion frames In this section, we present some perturbations of g-fusion frames and review some results about them. First, we need the following result proved in [3].

Lemma 1. Let $U$ be a Linear operator on a Banach space $X$ and assume that there exist $\lambda_{1}, \lambda_{2} \in[0,1)$, such that

$$
\|x-U x\| \leqslant \lambda_{1}\|x\|+\lambda_{2}\|U x\|
$$

for all $x \in X$. Then $U$ is bounded and invertible. Moreover,

$$
\frac{1-\lambda_{1}}{1+\lambda_{2}}\|x\| \leqslant\|U x\| \leqslant \frac{1+\lambda_{1}}{1-\lambda_{2}}\|x\|
$$

and

$$
\frac{1-\lambda_{2}}{1+\lambda_{1}}\|x\| \leqslant\left\|U^{-1} x\right\| \leqslant \frac{1+\lambda_{2}}{1-\lambda_{1}}\|x\|
$$

for all $x \in X$.

Theorem 8. Let $\Lambda:=\left(W_{j}, \Lambda_{j}, v_{j}\right)_{j \in \mathbb{J}}$ be a $g$-fusion frame for $H$ with bounds $A, B$ and $\left\{\Theta_{j} \in \mathcal{B}\left(H, H_{j}\right)\right\}_{j \in \mathbb{J}}$ be a sequence of operators, such that for any finite subset $\mathbb{I} \subseteq \mathbb{J}$ and for each $f \in H$ 


$$
\begin{array}{r}
\left\|\sum_{j \in \mathbb{I}} v_{j}^{2}\left(\pi_{W_{j}} \Lambda_{j}^{*} \Lambda_{j} \pi_{W_{j}} f-\pi_{W_{j}} \Theta_{j}^{*} \Theta_{j} \pi_{W_{j}} f\right)\right\| \leqslant \lambda\left\|\sum_{j \in \mathbb{I}} v_{j}^{2} \pi_{W_{j}} \Lambda_{j}^{*} \Lambda_{j} \pi_{W_{j}} f\right\|+ \\
+\mu\left\|\sum_{j \in \mathbb{I}} v_{j}^{2} \pi_{W_{j}} \Theta_{j}^{*} \Theta_{j} \pi_{W_{j}} f\right\|+\gamma\left(\sum_{j \in \mathbb{I}} v_{j}^{2}\left\|\Lambda_{j} \pi_{W_{j}} f\right\|^{2}\right)^{\frac{1}{2}}
\end{array}
$$

where $0 \leqslant \max \{\lambda+\gamma / \sqrt{A}, \mu\}<1$. Then $\Theta:=\left(W_{j}, \Theta_{j}, v_{j}\right)$ is a g-fusion frame for $H$ with bounds

$$
A \frac{1-\left(\lambda+\frac{\gamma}{\sqrt{A}}\right)}{1+\mu} \text { and } B \frac{1+\lambda+\frac{\gamma}{\sqrt{B}}}{1-\mu} .
$$

Proof. Assume that $\mathbb{I} \subseteq \mathbb{J}$ is a finite subset and $f \in H$. We have

$$
\begin{gathered}
\left\|\sum_{j \in \mathbb{I}} v_{j}^{2} \pi_{W_{j}} \Theta_{j}^{*} \Theta_{j} \pi_{W_{j}} f\right\| \leqslant\left\|\sum_{j \in \mathbb{I}} v_{j}^{2}\left(\pi_{W_{j}} \Lambda_{j}^{*} \Lambda_{j} \pi_{W_{j}} f-\pi_{W_{j}} \Theta_{j}^{*} \Theta_{j} \pi_{W_{j}} f\right)\right\|+ \\
+\left\|\sum_{j \in \mathbb{I}} v_{j}^{2} \pi_{W_{j}} \Lambda_{j}^{*} \Lambda_{j} \pi_{W_{j}} f\right\| \leqslant(1+\lambda)\left\|\sum_{j \in \mathbb{I}} v_{j}^{2} \pi_{W_{j}} \Lambda_{j}^{*} \Lambda_{j} \pi_{W_{j}} f\right\|+ \\
+\mu\left\|\sum_{j \in \mathbb{I}} v_{j}^{2} \pi_{W_{j}} \Theta_{j}^{*} \Theta_{j} \pi_{W_{j}} f\right\|+\gamma\left(\sum_{j \in \mathbb{I}} v_{j}^{2}\left\|\Lambda_{j} \pi_{W_{j}} f\right\|^{2}\right)^{\frac{1}{2}} .
\end{gathered}
$$

Then

$$
\begin{aligned}
\left\|\sum_{j \in \mathbb{I}} v_{j}^{2} \pi_{W_{j}} \Theta_{j}^{*} \Theta_{j} \pi_{W_{j}} f\right\| \leqslant \frac{1+\lambda}{1-\mu}\left\|\sum_{j \in \mathbb{I}} v_{j}^{2} \pi_{W_{j}} \Lambda_{j}^{*} \Lambda_{j} \pi_{W_{j}} f\right\|+ \\
\quad+\frac{\gamma}{1-\mu}\left(\sum_{j \in \mathbb{I}} v_{j}^{2}\left\|\Lambda_{j} \pi_{w_{j}} f\right\|^{2}\right)^{\frac{1}{2}} .
\end{aligned}
$$

Let $S_{\Lambda}$ be the g-fusion frame operator of $\Lambda$; then

$$
\left\|\sum_{j \in \mathbb{I}} v_{j}^{2} \pi_{W_{j}} \Lambda_{j}^{*} \Lambda_{j} \pi_{W_{j}} f\right\| \leqslant\left\|S_{\Lambda} f\right\| \leqslant B\|f\|,
$$

and also

$$
\begin{aligned}
\left\|\sum_{j \in \mathbb{I}} v_{j}^{2} \pi_{W_{j}} \Lambda_{j}^{*} \Lambda_{j} \pi_{W_{j}} f\right\| & =\sup _{\|g\|=1}\left|\left\langle\sum_{j \in \mathbb{I}} v_{j}^{2} \pi_{W_{j}} \Lambda_{j}^{*} \Lambda_{j} \pi_{W_{j}} f, g\right\rangle\right|= \\
& =\sup _{\|g\|=1}\left|\left\langle\sum_{j \in \mathbb{I}} v_{j} \Lambda_{j} \pi_{W_{j}} f, \Lambda_{j} \pi_{W_{j}} g\right\rangle\right| \leqslant
\end{aligned}
$$




$$
\begin{aligned}
& \leqslant\left(\sum_{j \in \mathbb{I}} v_{j}^{2}\left\|\Lambda_{j} \pi_{W_{j}} f\right\|^{2}\right)^{\frac{1}{2}}\left(\sum_{j \in \mathbb{I}} v_{j}^{2}\left\|\Lambda_{j} \pi_{W_{j}} f\right\|^{2}\right)^{\frac{1}{2}} \leqslant \\
& \leqslant \sqrt{B}\left(\sum_{j \in \mathbb{I}} v_{j}^{2}\left\|\Lambda_{j} \pi_{W_{j}} f\right\|^{2}\right)^{\frac{1}{2}} .
\end{aligned}
$$

Therefore, for all $f \in H$

$$
\begin{aligned}
&\left\|\sum_{j \in \mathbb{I}} v_{j}^{2} \pi_{W_{j}} \Theta_{j}^{*} \Theta_{j} \pi_{W_{j}} f\right\| \leqslant \\
& \leqslant\left(\frac{1+\lambda}{1-\mu} \sqrt{B}+\frac{\gamma}{1-\mu}\right)\left(\sum_{j \in \mathbb{I}} v_{j}^{2}\left\|\Lambda_{j} \pi_{W_{j}} f\right\|^{2}\right)^{\frac{1}{2}}<\infty .
\end{aligned}
$$

So, $\sum_{j \in \mathbb{J}} v_{j}^{2} \pi_{W_{j}} \Theta_{j}^{*} \Theta_{j} \pi_{W_{j}} f$ is unconditionally convergent. Let

$$
\begin{aligned}
S_{\Theta} & : H \longrightarrow H \\
S_{\Theta}(f) & =\sum_{j \in \mathbb{J}} v_{j}^{2} \pi_{W_{j}} \Theta_{j}^{*} \Theta_{j} \pi_{W_{j}} f .
\end{aligned}
$$

$S_{\Theta}$ is a well defined and bounded operator with

$$
\left\|S_{\Theta}\right\| \leqslant \frac{1+\lambda}{1-\mu} B+\frac{\gamma \sqrt{B}}{1-\mu}
$$

and for each $f \in H$. We have

$$
\sum_{j \in \mathbb{J}} v_{j}^{2}\left\|\Theta_{j} \pi_{W_{j}} f\right\|^{2}=\left\langle S_{\Theta} f, f\right\rangle \leqslant\left\|S_{\Theta}\right\|\|f\|^{2} .
$$

It follows that $\Theta:=\left(W_{j}, \Theta_{j}, v_{j}\right)_{j \in \mathbb{J}}$ is a g-fusion Bessel sequence for $H$. Thus, we obtain, by the hypothesis,

$$
\left.\left\|S_{\Lambda} f-S_{\Theta} f\right\| \leqslant \lambda\left\|S_{\Lambda} f\right\|+\mu\left\|S_{\Theta} f\right\|+\gamma\left(\sum_{j \in \mathbb{I}} v_{j}^{2}\left\|\Lambda_{j} \pi_{w_{j}} f\right\|\right)^{2}\right)^{\frac{1}{2}} .
$$

Therefore, by (3),

$$
\left\|f-S_{\Theta} S_{\Lambda}^{-1} f\right\| \leqslant \lambda\|f\|+\mu\left\|S_{\Theta} S_{\Lambda}^{-1} f\right\|+\gamma\left(\sum_{j \in \mathbb{J}} v_{J}^{2}\left\|\Lambda_{j} \pi_{W_{j}} S_{\Lambda}^{-1} f\right\|^{2}\right)^{\frac{1}{2}}
$$




$$
\leqslant\left(\lambda+\frac{\gamma}{\sqrt{A}}\right)\|f\|+\mu\left\|S_{\Theta} S_{\Lambda}^{-1} f\right\|
$$

Since $0 \leqslant \max \{\lambda+\gamma / \sqrt{A}, \mu\}<1$, then, by Lemma $1, S_{\Theta} S_{\Lambda}^{-1}$ and consequently $S_{\Theta}$ is invertible, and we get

$$
\left\|S_{\Theta}^{-1}\right\| \leqslant\left\|S_{\Lambda}^{-1}\right\|\left\|S_{\Lambda} S_{\Theta}^{-1}\right\| \leqslant \frac{1+\mu}{A\left(1-\left(\lambda+\frac{\gamma}{\sqrt{A}}\right)\right)} .
$$

So, the proof is completed.

Corollary 1. The optimal lower and upper bounds of $\Theta$ defined in Theorem 8 are $\left\|S_{\Theta}^{-1}\right\|^{-1}$ and $\left\|S_{\Theta}\right\|$, respectively.

Corollary 2. Let $\Lambda$ be a g-fusion frame for $H$ with bounds $A, B$ and $\left\{\Theta_{j} \in \mathcal{B}\left(H, H_{j}\right)\right\}_{j \in \mathbb{J}}$ be a sequence of operators. If there exists a constant $0<R<A$ such that

$$
\sum_{j \in \mathbb{J}} v_{j}^{2}\left\|\pi_{W_{j}} \Lambda_{j}^{*} \Lambda_{j} \pi_{W_{j}} f-\pi_{W_{j}} \Theta_{j}^{*} \Theta_{j} \pi_{W_{j}} f\right\| \leqslant R\|f\|
$$

for all $f \in H$, then $\Theta:=\left(W_{j}, \Theta_{j}, v_{j}\right)_{j \in \mathbb{J}}$ is a $g$-fusion frame for $H$ with bounds

$$
A-R \quad \text { and } \quad \min \left\{B+R \sqrt{\frac{B}{A}}, R+\sqrt{B}\right\} .
$$

Proof. It is easy to check that $\sum_{j \in \mathbb{J}} v_{j}^{2} \pi_{W_{j}} \Theta_{j}^{*} \Theta_{j} \pi_{W_{j}} f$ converges for any $f \in H$. Thus, we obtain, for each $f \in H$,

$$
\begin{aligned}
\sum_{j \in \mathbb{J}} v_{j}^{2}\left\|\pi_{W_{j}} \Lambda_{j}^{*} \Lambda_{j} \pi_{W_{j}} f-\pi_{W_{j}} \Theta_{j}^{*} \Theta_{j} \pi_{W_{j}} f\right\| & \leqslant R\|f\| \leqslant \\
& \leqslant \frac{R}{\sqrt{A}}\left(\sum_{j \in \mathbb{J}} v_{j}^{2}\left\|\Lambda_{j} \pi_{W_{j}} f\right\|^{2}\right)^{\frac{1}{2}}
\end{aligned}
$$

and also

$$
\begin{aligned}
\sum_{j \in \mathbb{J}} v_{j}^{2}\left\|\pi_{W_{j}} \Theta_{j}^{*} \Theta_{j} \pi_{W_{j}} f\right\| & \leqslant R\|f\|+\sum_{j \in \mathbb{J}} v_{j}^{2}\left\|\pi_{W_{j}} \Lambda_{j}^{*} \Lambda_{j} \pi_{W_{j}} f\right\| \leqslant \\
& \leqslant(R+\sqrt{B})\|f\| .
\end{aligned}
$$

By using Theorem 8 with $\lambda=\mu=0$ and $\gamma=\frac{R}{\sqrt{A}}$, the proof is completed. 
The following is another version of perturbation of g-fusion frames.

Theorem 9. Let $\Lambda$ be a $g$-fusion frame for $H$ with bounds $A, B$ and $\left\{\Theta_{j} \in \mathcal{B}\left(H, H_{j}\right)\right\}_{j \in \mathbb{J}}$ be a sequence of operators, such that for any finite subset $\mathbb{I} \subseteq \mathbb{J}$ and for each $\left\{f_{j}\right\}_{j \in \mathbb{J}} \in \mathscr{H}_{2}$,

$$
\begin{aligned}
\left\|\sum_{j \in \mathbb{I}} v_{j}\left(\pi_{W_{j}} \Lambda_{j}^{*} f_{j}-\pi_{W_{j}} \Theta_{j}^{*} f_{j}\right)\right\| \leqslant & \lambda\left\|\sum_{j \in \mathbb{I}} v_{j}\left(\pi_{W_{j}} \Lambda_{j}^{*} f_{j}\right)\right\|+ \\
& +\mu\left\|\sum_{j \in \mathbb{I}} v_{j}\left(\pi_{W_{j}} \Theta_{j}^{*} f_{j}\right)\right\|+\gamma\left(\sum_{j \in \mathbb{I}}\left\|f_{j}\right\|^{2}\right)^{\frac{1}{2}},
\end{aligned}
$$

where $0 \leqslant \max \{\lambda+\gamma / \sqrt{A}, \mu\}<1$. Then $\Theta:=\left(W_{j}, \Theta_{j}, v_{j}\right)_{j \in \mathbb{J}}$ is a g-fusion frame for $H$ with bounds

$$
A\left(\frac{1-\left(\lambda+\frac{\gamma}{\sqrt{A}}\right)^{2}}{1+\mu}\right) \text { and } B\left(\frac{1+\lambda+\frac{\gamma}{\sqrt{B}}}{1-\mu}\right)^{2} .
$$

Proof. Let $\left\{f_{j}\right\}_{j \in \mathbb{J}} \in \mathscr{H}_{2}$; then

$$
\begin{gathered}
\left\|\sum_{j \in \mathbb{I}} v_{j} \pi_{W_{j}} \Theta_{j}^{*} f_{j}\right\| \leqslant\left\|\sum_{j \in \mathbb{I}} v_{j}\left(\pi_{W_{j}} \Lambda_{j}^{*} f_{j}-\pi_{W_{j}} \Theta_{j}^{*} f_{j}\right)\right\|+\left\|\sum_{j \in \mathbb{I}} v_{j} \pi_{W_{j}} \Lambda_{j}^{*} f_{j}\right\| \leqslant \\
\leqslant(1+\lambda)\left\|\sum_{j \in \mathbb{I}} v_{j} \pi_{W_{j}} \Lambda_{j}^{*} f_{j}\right\|+\mu\left\|\sum_{j \in \mathbb{I}} v_{j} \pi_{W_{j}} \Theta_{j}^{*} f_{j}\right\|+\gamma\left(\sum_{j \in \mathbb{I}}\left\|f_{j}\right\|^{2}\right)^{\frac{1}{2}}
\end{gathered}
$$

Hence,

$$
\begin{aligned}
\left\|\sum_{j \in \mathbb{I}} v_{j} \pi_{W_{j}} \Theta_{j}^{*} f_{j}\right\| & \leqslant \frac{1+\lambda}{1-\mu}\left\|\sum_{j \in \mathbb{I}} v_{j} \pi_{W_{j}} \Lambda_{j}^{*} f_{j}\right\|+\frac{\gamma}{1-\mu}\left(\sum_{j \in \mathbb{I}}\left\|f_{j}\right\|^{2}\right)^{\frac{1}{2}} \leqslant \\
& \leqslant\left(\frac{1+\lambda}{1-\mu} \sqrt{B}+\frac{\gamma}{1-\mu}\right)\left(\sum_{j \in \mathbb{I}}\left\|f_{j}\right\|^{2}\right)^{\frac{1}{2}} .
\end{aligned}
$$

Let

$$
\begin{gathered}
T_{\Theta}: H \longrightarrow \mathscr{H}_{2}, \\
T_{\Theta}\left\{f_{j}\right\}_{j \in \mathbb{I}}=\sum_{j \in \mathbb{I}} v_{j} \pi_{W_{j}} \Theta_{j}^{*} f_{j} .
\end{gathered}
$$

Therefore, $T_{\Theta}$ is well-defined and bounded. Then, by Theorem $1, \Theta$ is a g-fusion Bessel sequence. Suppose that $G:=T_{\Theta} T_{\Lambda}^{*} S_{\Lambda}^{-1}$. Then we get, by 
the hypothesis and (4) for $f_{j}:=\Lambda_{j} \pi_{W_{j}} S_{\Lambda}^{-1} f$,

$$
\begin{aligned}
\|f-G f\| & \leqslant \lambda\|f\|+\mu\|G f\|+\gamma\left(\sum_{j \in \mathbb{J}} v_{j}^{2}\left\|\Lambda_{j} \pi_{W_{j}} S_{\Lambda}^{-1} f\right\|^{2}\right)^{\frac{1}{2}} \leqslant \\
& \leqslant\left(\lambda+\frac{\gamma}{\sqrt{A}}\right)\|f\|+\mu\|G f\| .
\end{aligned}
$$

Since $0 \leqslant \max \left\{\lambda+\frac{\gamma}{\sqrt{A}}, \mu\right\}<1$, by Lemma $1, G$ and, consequently, $T_{\Theta} T_{\Lambda}^{*}$ are invertible and

$$
\left\|G^{-1}\right\| \leqslant \frac{1+\mu}{1-\left(\lambda+\frac{\gamma}{\sqrt{A}}\right)} .
$$

Now, let $f \in H$, and we have

$$
\begin{gathered}
\|f\|^{4}=\left|\left\langle G G^{-1} f, f\right\rangle\right|^{2}=\left|\left\langle\sum_{j \in \mathbb{J}} v_{j}^{2} \pi_{W_{j}} \Theta_{j}^{*} \Lambda_{j} \pi_{W_{j}} S_{\Lambda}^{-1}\left(G^{-1} f\right), f\right\rangle\right|^{2} \leqslant \\
\leqslant \sum_{j \in \mathbb{J}}\left\|\Lambda_{j} \pi_{W_{j}} S_{\Lambda}^{-1}\left(G^{-1} f\right)\right\|^{2} \cdot \sum_{j \in \mathbb{J}} v_{j}^{2}\left\|\Theta_{j} \pi_{W_{j}} f\right\|^{2} \leqslant \\
\leqslant A^{-1}\left\|G^{-1} f\right\|^{2} \sum_{j \in \mathbb{J}} v_{j}^{2}\left\|\Theta_{j} \pi_{W_{j}} f\right\|^{2} .
\end{gathered}
$$

This completes the proof.

Theorem 10. Let $\Lambda$ be a g-fusion frame for $H$ with bounds $A, B$ and $\left\{\Theta_{j} \in \mathcal{B}\left(H, H_{j}\right)\right\}_{j \in \mathbb{J}}$ be a sequence of operators. If there exists a constant $0<R<A$ such that

$$
\left.\sum_{j \in \mathbb{I}} v_{j}^{2} \| \Lambda_{j} \pi_{W_{j}} f-\Theta_{j} \pi_{W_{j}} f\right)\left\|^{2} \leqslant R\right\| f \|^{2}
$$

for all $f \in H$, then $\Theta:=\left(W_{j}, \Theta_{j}, v_{j}\right)_{j \in \mathbb{J}}$ is a $g$-fusion frame for $H$ with bounds

$$
(\sqrt{A}-\sqrt{R})^{2} \quad \text { and } \quad(\sqrt{R}+\sqrt{B})^{2} .
$$

Proof. Let $f \in H$. We can write

$$
\begin{aligned}
\left\|\left\{v_{j} \Theta_{j} \pi_{W_{j}} f\right\}_{j \in \mathbb{J}}\right\|_{2} \leqslant\left\|\left\{v_{j} \Theta_{j} \pi_{W_{j}} f-v_{j} \Lambda_{j} \pi_{W_{j}} f\right\}_{j \in \mathbb{J}}\right\|_{2}+ & \\
& +\left\|\left\{v_{j} \Lambda_{j} \pi_{W_{j}} f\right\}_{j \in \mathbb{J}}\right\|_{2} \leqslant(\sqrt{R}+\sqrt{B})^{2}\|f\|^{2}
\end{aligned}
$$

and also

$\left\|\left\{v_{j} \Theta_{j} \pi_{W_{j}} f\right\}_{j \in \mathbb{J}}\right\|_{2} \geqslant\left\|\left\{v_{j} \Lambda_{j} \pi_{W_{j}} f\right\}_{j \in \mathbb{J}}\right\|_{2}-\left\|\left\{v_{j} \Theta_{j} \pi_{W_{j}} f-v_{j} \Lambda_{j} \pi_{W_{j}} f\right\}_{j \in \mathbb{J}}\right\|_{2} \geqslant$ 


$$
\geqslant(\sqrt{A}-\sqrt{R})^{2}\|f\|^{2}
$$

This complete the proof.

\section{References}

[1] Blocsli H., Hlawatsch H. F., Fichtinger H. G. Frame-theoretic analysis of oversampled filter bank. IEEE Trans. Signal Processing. Anal., 1998, vol. 46, no. 12, pp. 3256 - 3268. DOI: https://doi.org/10.1109/78.735301.

[2] Candes E. J., Donoho D. L. New tight frames of curvelets and optimal representation of objects with piecewise $C^{2}$ singularities. Comm. Pure and App. Math. Anal., 2004, vol. 57, no. 2, pp. 219-266.

DOI: https://doi.org/10.1002/cpa.10116.

[3] Casazza P. G., Christensen O. Perturbation of operators and application to frame theory. J. Fourier Anal. Appl. Anal., 1997, vol. 3, pp. 543-557. DOI: https://doi.org/10.1007/BF02648883.

[4] Casazza P. G., Kutyniok G. Frames of subspaces. Contemp. Math. Anal., 2004, vol. 345, pp. $87-114$.

[5] Casazza P. G., Kutyniok G., Li S. Fusion frames and distributed processing. Appl. Comput. Harmon. Anal., 2008, vol. 25, no. 1, pp. 114-132.

DOI: https://doi.org/10.1016/j.acha.2007.10.001.

[6] Christensen O. Frames and Riesz bases (An introduction course). Birkhäuser, 2008.

[7] Diestel J. Sequences and series in Banach spaces. Springer-Verlag, New York, 1984.

[8] Duffin R. J., Schaeffer A. C. A class of nonharmonic Fourier series. Trans. Amer. Math., vol. 72, no. 1, pp. 341-366.

[9] Faroughi M. H., Ahmadi R. Some properties of c-frames of subspaces. J. Nonlinear Sci. Appl. Anal., 2008, vol. 1, no. 3, pp. 155-168.

[10] Feichtinger H. G., Werther T. Atomic systems for subspaces. Proceedings SampTA. Orlando, FL Anal., 2001, pp. 163-165.

[11] Găvruţa L. Frames for operators. Appl. Comp. Harm. Anal., 2012, vol. 32, pp. 139 -144. DOI: http://dx.doi.org/10.1016/j.acha.2011.07.006.

[12] Heuser H. Functional analysis. John Wiley, New York, 1991.

[13] Khayyami M., and Nazari A. Construction of continuous g-frames and continuous fusion frames. SCMA. Anal., 2016, vol. 4, no. 1, pp. 43-55.

[14] Najati A., Faroughi M. H., Rahimi A. G-frames and stability of g-frames in Hilbert spaces. Method Func. Anal. Top., 2008, vol. 14, no. 3, pp. 271-286. 
[15] Sun W. G-Frames and G-Riesz bases. J. Math. Anal. Appl., 2006, 322, pp. 437 -452. DOI: https://doi.org/10.1016/j.jmaa.2005.09.039.

Received July 30, 2019.

In revised form, December 13, 2019.

Accepted December 28, 2019.

Published online January 15, 2020.

Gh. Rahimlou

Department of Mathematics, Faculty of Tabriz Branch, Technical and Vocational University (TVU), East Azarbaijan, Iran.

E-mail: grahimlou@gmail.com

V. Sadri

Department of Mathematics, Faculty of Tabriz Branch, Technical and Vocational University (TVU), East Azarbaijan, Iran.

E-mail:vahidsadri57@gmail.com

R. Ahmadi

Institute of Fundamental Sciences, University of Tabriz, Iran.

E-mail:rahmadi@tabrizu.ac.ir 